\title{
Self-Similar Cylindrical Ionizing Shock Waves in a Non-Ideal Gas with Radiation Heat-Flux
}

\author{
J. P. Vishwakarma*, Mahendra Singh \\ Dept. of Mathematics and Statistics, D.D.U. Gorakhpur University Gorakhpur, 273009, India
}

\begin{abstract}
Self-similar flows behind a gas-ionizing cylindrical shock wave, with radiation heat flux, in a non-ideal gas are studied. The ionizing shock is assumed to be propagating in a medium at rest with constant density permeated by an azimuthal magnetic field. The electrical conductivity of the gas is infinite behind shock and zero ahead of it. Effects of the non-idealness of the gas, the radiation flux and the rate of energy input from the inner contact surface (or piston) on the flow-field behind the shock and on the shock propagation are investigated.
\end{abstract}

Keywords IonizingShock Wave, Non-Ideal Gas, Spatially Variable Magnetic Field, Similarity Solutions, Radiation Heat-Flux

\section{Introduction}

The influence of radiation on a shock wave and on the flow-field behind the shock front has always been of great interest, for instance, in the field of nuclear power and space research. Consequently, similarity models for classical blast wave problems have been extended by taking radiation into account (Elliott[1], Wang[2], Helliwell[3], Ni Castro[4], Ghoniem et al.[5]). Elliott[1] considered the explosion problem by introducing the radiation flux in its diffusion approximation. Wang[2] discussed the piston problem with radiative heat transfer in the thin and thick limits and also in the general case with idealized two direction approximation. Ghoniem et al.[5] obtained a self-similar solution for spherical explosion taking into account the effects of both conduction and radiation in the two limits of Rosselandradiative diffusion and Plank radiative emission.

Since at high temperatures that prevail in the problems associated with shock waves a gas is partially ionized, electromagnetic effects may also be significant. A complete analysis of such a problem should therefore consist of the study of the gasdynamic flow and the electromagnetic and radiation fields simultaneously. A number of problem relating to shock wave propagation in a perfect gas with radiative and magnetohydrodynamic effects have been studied (see, for example, Singh and Mishra[6], Vishwakarma et al.[7], Singh et al.[8], Ganguly and Jana[9]).

The assumption that the gas is ideal is no more valid when the flow takes place at extreme conditions. In recent years,

\footnotetext{
* Corresponding author:
}

jpv_univgkp@yahoo.com (J. P. Vishwakarma)

Published online at http://journal.sapub.org/am

Copyright (C) 2012 Scientific \& Academic Publishing. All Rights Reserved several studies have been performed concerning the problem of strong shock waves in non-ideal gases, in particular, by Anisimov and Spiner[10], Rangarao and Purohit[11], Wu and Roberts[12], Madhumita and Sharma[13], Arora and Sharma[14] and Vishwakarma and Nath[15,16] among others. Anisimov and Spiner[10] have taken an equation of state for a low density non-ideal gas in a simplified form, and studied the effects of non-idealness of the gas on the problem of point explosion. Rangarao and Purohit[11] have studied the self-similar flows of a non-ideal gas driven by an expanding piston and obtained approximate analytic and numerical solutions by taking the equation of state suggested by Anisimov and Spiner[10]. Vishwakarma and Nath[15] have obtained similarity solutions for the flow behind an exponential shock by taking the same equation of state for the medium. Roberts and $\mathrm{Wu}[17,18]$ have used an equivalent equation of state to study the shock wave theory of sonoluminescence. Vishwakarma et al.[19] too adopted this as their model of a non-ideal gas to obtain the self-similar solutions for the flow behind a magnetogasdynamic cylindrical shock wave propagating in a rotating gas in presence of an azimuthal magnetic field. They have taken the electrical conductivity of the initial medium and the medium behind the shock to be infinite. But, in many practical cases the medium may be of low conductivity which becomes highly conducting due to passage of a strong shock. Such a shock wave is called a gas-ionizing shock or, simply ionizing shock. The propagation of ionizing shock has been studied by Greenspan[20], Greifinger and Cole[21], Christer[22], Rangarao and Ramana[23] and Singh[24] in a perfect gas.

We, in the present work, extended the problem treated by Singh[24] by taking the medium to be a non-ideal gas instead of an ideal gas. The problem of line explosion with time dependent energy release and radiation in the presence of an 
azimuthal magnetic field is considered due to its relevance to the experiments on pinch effect and exploding wires. A gas-ionizing cylindrical shock wave, generated by line explosion, is propagated in the non-ideal gas. The counter pressure (the pressure ahead of the shock) is taken into account. The radiation pressure and radiation energy are considered very small in comparison to material pressure and energy, respectively, and therefore only radiation flux is taken into account. A diffusion model for an optically thick grey gas is assumed. The ambient azimuthal magnetic field is assumed to be varying as some power of the distance from the axis of symmetry. In order to obtain similarity solutions of the problem it is necessary to take the density of the ambient non-ideal gas to be a constant. It is observed that the shock velocity decreases with the ambient magnetic field. Also, the rate of energy input to the flow behind the shock, the radiation flux and the non-idealness of the gas are found to have significant effects on the propagation of the shock and the flow-field behind it.

\section{Basic Equations and Boundary Conditions}

The fundamental equations governing the unsteady and cylindrically symmetric motion of an inviscid, perfectly conducting and non-ideal gas in which the effects of radiation flux and azimuthal magnetic field may be significant, can be written as (Christer and Helliwell[25], Vishwakarma and Pandey[26], Gretler and Wehle[27]).

$$
\begin{gathered}
\frac{\mathrm{d} \rho}{\mathrm{dt}}+\frac{\rho}{\mathrm{r}} \frac{\partial}{\partial \mathrm{r}}(\mathrm{r} \mathrm{u})=0, \\
\frac{\mathrm{du}}{\mathrm{dt}}+\frac{1}{\rho} \frac{\partial \mathrm{p}}{\partial \mathrm{r}}+\frac{\mathrm{h}}{\rho \mathrm{r}} \frac{\partial}{\partial \mathrm{r}}(\mathrm{rh})=0, \\
\frac{\mathrm{dh}}{\mathrm{dt}}+\mathrm{h} \frac{\partial \mathrm{u}}{\partial \mathrm{r}}=0, \\
\frac{\mathrm{de}}{\mathrm{dt}}+\mathrm{p} \frac{\mathrm{d}}{\mathrm{dt}}\left(\frac{1}{\rho}\right)+\frac{1}{\rho \mathrm{r}} \frac{\partial}{\partial \mathrm{r}}(\mathrm{rq})=0,
\end{gathered}
$$

where $\frac{\mathrm{d}}{\mathrm{dt}}=\frac{\partial}{\partial \mathrm{t}}+\mathrm{u} \frac{\partial}{\partial \mathrm{r}}$

Here, $\rho$ is the density, $p$ the pressure, $u$ the radial velocity, $\mathrm{h}$ the azimuthal magnetic field, $\mathrm{q}$ the radiation heat flux, $\mathrm{t}$ the time, $r$ the distance from the axis of symmetry, and e the internal energy. The magnetic permeability of the medium is taken to be unity.

In most of the cases the propagation of shock waves arise in extreme conditions under which the assumption that the gas is ideal is not a sufficiently accurate description. To discover how deviations from the ideal gas can affect the solutions, we adopt a simple model. We assume that the gas obeys a simplified van der Waals equation of state of the form (Roberts and $\mathrm{Wu},[17,18]$ )

$$
\mathrm{p}=\frac{\Gamma \rho \mathrm{T}}{(1-\mathrm{b} \rho)}
$$

$$
\mathrm{e}=\mathrm{C}_{\mathrm{v}} \mathrm{T}=\frac{\mathrm{p}(1-\mathrm{b} \rho)}{\rho(\gamma-1)}
$$

where $\Gamma$ is the gas constant, $\mathrm{C}_{\mathrm{v}}=\frac{\Gamma}{(\gamma-1)}$ is the specific heat at constant volume and $\gamma$ is the ratio of specific heats. The constant $b$ is the 'van der Waals excluded volume'; it places a limit, $\rho_{\max }=\frac{1}{b}$, on the density of the gas.

For an isentropic change of state of the non-ideal gas, we may calculate the so called speed of sound in non-ideal gas as follows

$$
a=\left(\frac{d p}{d \rho}\right)_{s}^{1 / 2}=\left[\frac{\gamma p}{\rho(1-b \rho)}\right]^{1 / 2},
$$

where the subscript 's' refers to the process of constant entropy.

Assuming local thermodynamic equilibrium and a diffusion model for an optically thick grey gas (Pomraning[28]), the differential approximation of the radiation transport equation can be written in the following form

$$
q=-\frac{c \mu}{3} \frac{\partial}{\partial r}\left(\sigma T^{4}\right)
$$

where $\frac{1}{4} \sigma c$ is the Stefan-Boltzmann constant, c the velocity of light and $\mu$ the Rosseland mean free path for radiation. The assumption of an optically thick grey gas is physically consistent with the neglect of radiation pressure and radiation energy in the equation system (2.1)-(2.4) (Ni-Castro [4]).

Following Wang[2], we take

$$
\mu=\mu_{0} \rho^{\alpha} T^{\chi},
$$

where $\mu_{0}, \alpha$ and $\chi$ are constants. It will be seen later that the exponents $\alpha$ and $\chi$ must satisfy the similarity requirements. The self-similarity condition puts no constraints on specification of the density dependence of $\mu$.

We assume that a cylindrical shock is propagating in the medium and the flow variables immediately ahead of the shock front are

$$
\begin{gathered}
\mathrm{u}=0, \\
\rho=\rho_{1}=\text { constant }, \\
\mathrm{h}=\mathrm{h}_{1}=\mathrm{AR}^{\mathrm{n}}(\mathrm{n}<0) .(\text { Rosenou }[29]), \\
\mathrm{p}=\mathrm{p}_{1}=\frac{-(\mathrm{n}+1)}{2 \mathrm{n}} \mathrm{A}^{2} \mathrm{R}^{2 \mathrm{n}},
\end{gathered}
$$

$\mathrm{q}=\mathrm{q}_{1}=0$ (Laumbach and Probstein [30]), (2.10e)

where $\mathrm{R}$ is the shock radius, and $\mathrm{A}$ and $\mathrm{n}$ are constants. Due to passes of the shock, the gas is highly ionized and its electrical conductivity becomes infinitely large. The conditions across such a gas-ionizing shock are (c.f. Singh and Srivastava[31] and Vishwakarma and Pandey[26])

$$
\begin{gathered}
\rho_{2}\left(V-u_{2}\right)=\rho_{1} V=m_{s}, \\
p_{2}-p_{1}=m_{s} u_{2}, \\
e_{2}+\frac{p_{2}}{\rho_{2}}+\frac{1}{2}\left(V-u_{2}\right)^{2}-\frac{q_{2}}{m_{s}}=e_{1}+\frac{p_{1}}{\rho_{1}}+\frac{1}{2} V^{2},
\end{gathered}
$$




$$
\mathrm{h}_{2}=\mathrm{h}_{1} \text {, }
$$

where subscripts 2 and 1 are for the regions just behind and just ahead of the shock surface respectively, and $\mathrm{V}$ denotes the shock velocity. The shock front is assumed to be opaque and it does not receive any heat flux from external sources. Therefore, the heat flux $\mathrm{q}_{2}$ is the heat flux exchanged between the flow-field and the shock front. The jump conditions (2.11) are not sufficient to determine all the flow variables at the shock front. Hence, one variable stays undetermined there. This difficulty is removed by assuming the shock front to isothermal that is,

$$
\mathrm{T}_{1}=\mathrm{T}_{2}
$$

(Zel'dovich and Raizer[32], Rosenau and Frankenthal[33], Singh and Mishra[6]).

The shock conditions (2.11) may be written as

$$
\begin{aligned}
& \rho_{2}=\frac{\rho_{1}}{\beta}, \\
& \mathrm{u}_{2}=(1-\beta) \mathrm{V} \text {, } \\
& \mathrm{p}_{2}=\rho_{1} \mathrm{~V}^{2}\left[\frac{\mathrm{p}_{1}}{\rho_{1} \mathrm{~V}^{2}}+(1-\beta)\right] \text {, } \\
& \mathrm{q}_{2}=\frac{\rho_{1} \mathrm{~V}^{3}(\gamma+1)}{2(\gamma-1)}\left[\begin{array}{c}
\frac{2 \beta}{(\gamma+1)}\left(\frac{1}{\mathrm{M}^{2}}+\gamma+\overline{\mathrm{b}}\right)-\beta^{2} \\
-\frac{2 \overline{\mathrm{b}}}{(\gamma+1)}-\frac{2}{\mathrm{M}^{2}(\gamma+1)}-\frac{(\gamma-1)}{\gamma+1}
\end{array}\right], \\
& \mathrm{h}_{2}=\mathrm{h}_{1} \text {, } \\
& \text { Where } \beta=\overline{\mathrm{b}}+\frac{1}{\gamma \mathrm{M}^{2}} \text {, }
\end{aligned}
$$

and $\overline{\mathrm{b}}=\mathrm{b} \rho_{1}$ is the parameter of non-idealness of the gas. Here, $M$ is the shock-Mach number referred to the frozen speed of sound $\left(\frac{\gamma \mathrm{p}_{1}}{\rho_{1}}\right)^{1 / 2}$, and $\mathrm{M}_{\mathrm{A}}$ the Alfven-Mach number.

The shock-Mach number $M_{e}$ referred to the speed of sound in non-ideal gas $\left(\frac{\gamma \mathrm{p}_{1}}{\rho_{1}\left(1-\mathrm{b} \rho_{1}\right)}\right)^{1 / 2}$ and the Alfven-Mach number $\mathrm{M}_{\mathrm{A}}$ are given by

$$
M_{e}=M\left(1-b \rho_{1}\right)^{1 / 2} \text { and } M_{A}=\frac{V}{\left(h_{1}^{2} / \rho_{1}\right)^{1 / 2}},
$$

where $\quad M=\frac{V}{\left(\gamma \mathrm{p}_{1} / \rho_{1}\right)^{1 / 2}}$.

The total energy of the flow-field behind the shock is not constant, but assumed to be time dependent and varying as (Rogers[34], Freeman[35])

$$
\mathrm{E}=\mathrm{E}_{0} \mathrm{t}^{\omega}, \omega \geq 0
$$

The positive values of $\omega$ correspond to the class in which the total energy increases with time. This increase can be achieved by the pressure exerted on the fluid by an expanding surface (a contact surface or a piston). This surface may be, physically, the surface of the stellar corona or the condensed explosives or the diaphragm containing a very high-pressure driver gas. By sudden expansion of the stellar corona or the detonation products or the driver gas into the ambient gas, a shock wave is produced in the ambient gas. The shocked gas is separated from this expanding surface which is a contact discontinuity. This contact surface acts as a 'piston' for the shock wave. Thus the flow is headed by a shock front and has an expanding surface as the inner boundary. A situation very much of the same kind may prevail during the formation of a cylindrical spark channel from exploding wires. In addition, in the usual cases of spark break down, time-dependent energy input is a more realistic assumption than instantaneous energy input (Freeman and Cragges [36], Director and Dabora [37]).

The expression for the total energy of the non-ideal gas behind the shock is given by

$$
\mathrm{E}=2 \pi \int_{\mathrm{r}_{\mathrm{p}}}^{\mathrm{R}}\left[\frac{1}{2} \rho \mathrm{u}^{2}+\frac{\mathrm{p}(1-\mathrm{b} \rho)}{(\gamma-1)}+\frac{1}{2} \mathrm{~h}^{2}\right] \mathrm{rdr}=\mathrm{E}^{0} \mathrm{t}^{\omega},
$$

where $r_{p}$ is the position of inner contact surface.

\section{Similarity Solutions}

For self-similar motions, the system of partial differential equations (2.1)-(2.4), (2.8) reduces to a system of ordinary differential equations in new unknown functions of the similarity variable $\eta=r / R$. Let us derive these equations. To do this we represent the solution of the partial differential equations (2.1)-(2.4), (2.8) in terms of the products of scale functions and the new unknown functions of the similarity variable $\eta, \eta=r / R, R=R(t)$. The pressure, density, velocity, magnetic field, radiation heat flux, and length scales are not all independent of each other. If we choose $R$ and $\rho_{1}$ as the basic scales, then the quantity $\mathrm{V}=\dot{\mathrm{R}}$ can serve as the velocity scale, $\rho_{1} \mathrm{~V}^{2}$ as the pressure scale, $\rho_{1}^{1 / 2} \mathrm{~V}$ as the magnetic field scale, and $\rho_{1} \mathrm{~V}^{3}$ as the radiation flux scale. This does not limit the generality of the solution, as the scale is only defined to within a numerical coefficient which can always be included in the new unknown function. We seek a solution of the form (Abdel-Raouf and Gretler[38], Ghoniem et al.[5])

$$
\begin{aligned}
& u=V U(\eta), \\
& \rho=\rho_{1} D(\eta), \\
& p=\rho_{1} V^{2} P(\eta), \\
& h=\rho_{1}^{1 / 2} V H(\eta), \\
& q=\rho_{1} V^{3} Q(\eta),
\end{aligned}
$$

where $\mathrm{U}, \mathrm{D}, \mathrm{P}, \mathrm{H}$, and $\mathrm{Q}$ are the functions of the non-dimensional variable (similarity variable) $\eta$ only.

Applying the similarity transformations (3.1) to (3.5) to the relation (2.15), we find that the motion of the shock front is given by the equation

$$
\begin{gathered}
\mathrm{R}^{2} \mathrm{~V}^{2}=\frac{\mathrm{E}_{0} \mathrm{t}^{\omega}}{2 \pi \rho_{\mathrm{l}} \mathrm{J}}, \\
\text { where } \mathrm{J}=\int_{\eta_{\mathrm{p}}}^{1}\left[\frac{1}{2} \mathrm{D} \mathrm{U}^{2}+\frac{\mathrm{P}(1-\overline{\mathrm{b}} \mathrm{D})}{(\gamma-1)}+\frac{\mathrm{H}^{2}}{2}\right] \eta \mathrm{d} \eta
\end{gathered}
$$


Equation (3.6) can be written as

$$
\mathrm{R} \frac{\mathrm{dR}}{\mathrm{dt}}=\left(\frac{\mathrm{E}_{0}}{2 \pi \rho_{1} \mathrm{~J}}\right)^{1 / 2} \mathrm{t}^{\mathrm{\omega} / 2},
$$

which on integration, gives

$$
\mathrm{R}=\left(\frac{8 \mathrm{E}_{0}}{\pi \rho_{1} \mathrm{~J}}\right)^{1 / 4} \frac{1}{\sqrt{(\omega+2)}} \mathrm{t}^{(\omega+2) / 4}
$$

From (3.9), we get the shock velocity

$$
\mathrm{V}=\frac{\mathrm{dR}}{\mathrm{dt}}=\frac{(\omega+2) \mathrm{R}}{4 \mathrm{t}}=\mathrm{KR}^{(\omega-2) /(\omega+2)}
$$

where $\mathrm{K}=\left(\frac{8 \mathrm{E}_{0}}{\pi \rho_{1} \mathrm{~J}}\right)^{1 / \omega+2} \frac{(\omega+2)^{\omega / \omega+2}}{4}$.

Since $M$ and $M_{A}$ are constants for similarity solutions, we have

$$
\mathrm{n}=\frac{\omega-2}{\omega+2}, \quad(0<\omega<2)
$$

Thus, the shock velocity $\mathrm{V}$ decreases as the ambient magnetic field $h_{1}$.

The conservation equations (2.1) to (2.4) can be transformed into following system of ordinary differential equations with respect to $\eta$ :

$$
\begin{gathered}
\frac{\mathrm{dD}}{\mathrm{d} \eta}=\left[\frac{\mathrm{U}+\eta \frac{\mathrm{dU}}{\mathrm{d} \eta}}{\eta(\eta-\mathrm{U})}\right] \mathrm{D}, \\
\frac{\mathrm{dH}}{\mathrm{d} \eta}=\left[\frac{\eta+\frac{\mathrm{dU}}{\mathrm{d} \eta}}{\eta-\mathrm{U}}\right] \mathrm{H}, \\
\frac{\mathrm{dP}}{\mathrm{d} \eta}=\left[\mathrm{D}\left\{(\eta-\mathrm{U}) \frac{\mathrm{dU}}{\mathrm{d} \eta}-\mathrm{nU}\right\}-\mathrm{H}^{2}\left(\frac{1}{\eta}+\frac{\frac{\mathrm{dH}}{\mathrm{d} \eta}}{\mathrm{H}}\right)\right], \\
\frac{\mathrm{dQ}}{\mathrm{d} \eta}=\left[\frac{\mathrm{dP}}{\mathrm{d} \eta}(\eta-\mathrm{U}) \frac{(1-\overline{\mathrm{b}} \mathrm{D})}{(\gamma-1)}-\frac{2 \mathrm{n}(1-\overline{\mathrm{b}} \mathrm{D}) \mathrm{P}}{(\gamma-1)}\right] \\
+\left[-\frac{\mathrm{Q}}{\eta}-\frac{\mathrm{dD}}{\mathrm{d} \eta} \mathrm{D}\left\{\gamma(\eta-\mathrm{U})-\frac{1}{(\gamma-1)}\right\} \mathrm{P}\right]
\end{gathered}
$$

By using equations (2.5) and (2.9) in (2.8), we get

$$
\mathrm{q}=-\frac{4}{3} \mathrm{c} \mu_{0} \sigma \rho^{\alpha} \frac{\{\mathrm{p}(1-\mathrm{b} \rho)\}^{3+\chi}}{\Gamma^{3+\chi} \rho^{3+\chi}} \frac{\partial \mathrm{T}}{\partial \mathrm{r}}
$$

Again, using the equation (2.5) in (3.16), and then the similarity transformations (3.2), (3.3) and (3.5), we get

$$
\begin{gathered}
\mathrm{Q}=-\frac{4}{3} \frac{\mu_{0} \sigma \mathrm{c}}{\Gamma^{4+\chi}} \rho_{1}^{\alpha-1} \mathrm{~K}^{5+2 \chi}(1-\overline{\mathrm{b}} \mathrm{D})^{3+\chi} \mathrm{D}^{\alpha-\chi-4} \mathrm{P}^{4+\chi} \mathrm{R}^{5 \mathrm{n}+2 \mathrm{n} \chi-1} \\
{\left[\frac{\mathrm{P}^{\prime}}{\mathrm{P}}(1-\overline{\mathrm{b}} \mathrm{D})-\frac{\mathrm{D}^{\prime}}{\mathrm{D}}\right]}
\end{gathered}
$$

Equation (3.17) shows that the similarity solution of the present problem exists only when

$$
5 \mathrm{n}+2 \mathrm{n} \chi-1=0 \text {. }
$$

Therefore, $\chi=\frac{6-2 \omega}{\omega-2}$.

Therefore, equation (3.17) becomes

$$
\begin{aligned}
& Q=-N(1-\bar{b} D)^{3+\chi} D^{\alpha-\chi}{ }^{-4} P^{4+\chi}\left[\frac{P^{\prime}}{P}(1-\bar{b} D)-\frac{D^{\prime}}{D}\right], \\
& \text { where } N=\frac{4}{3} \frac{\mu_{0} \sigma c}{\Gamma^{4+\chi}} \rho_{1}^{\alpha-1} K^{5+2 \chi}
\end{aligned}
$$

is a non-dimensional radiation parameter. $\mathrm{N}$ depends on the mean free path of radiation.

By using (3.12) and (3.14) in (3.18), we get

$$
\begin{gathered}
\frac{\mathrm{dU}}{\mathrm{d} \eta} \frac{\frac{1}{\left\{\left((\eta-\mathrm{U}) \frac{\mathrm{D}}{\mathrm{P}}-\frac{\mathrm{H}^{2}}{\mathrm{P}(\eta-\mathrm{U})}\right)(1-\overline{\mathrm{b}} \mathrm{D})-\frac{1}{\eta-\mathrm{U}}\right\}}\left[\left\{\frac{1}{\eta(\eta-\mathrm{U})}+\right.\right.}{} \\
\left(\frac{\mathrm{nD}}{\mathrm{P}}+\frac{1}{\mathrm{U}}\left(\frac{1}{\eta}+\frac{\mathrm{n}}{(\eta-\mathrm{U})}\right) \frac{\mathrm{H}^{2}}{\mathrm{P}}\right)(1-\overline{\mathrm{b}} \mathrm{D}) \\
-\frac{\mathrm{Q}}{\left.\left.\mathrm{N}(1-\overline{\mathrm{b}} \mathrm{D})^{3+\chi} \mathrm{D}^{\alpha-\chi+4} \mathrm{P}^{\chi+4}\right\}\right] \mathrm{U}}
\end{gathered}
$$

Using the self-similarity transformations (3.1) to (3.5), equations (2.12) can be rewritten as

$$
\begin{gathered}
\mathrm{U}(1)=1-\beta, \\
\mathrm{P}(1)=1-\overline{\mathrm{b}}, \\
\mathrm{D}(1)=\frac{1}{\beta}, \\
\mathrm{H}(1)=\left[\begin{array}{c}
\mathrm{H}^{2}(1) \\
\frac{\beta}{(\gamma-1)}\left(\gamma+\overline{\mathrm{b}}+\frac{1}{\mathrm{M}^{2}}\right)-\frac{(\gamma+1)}{2(\gamma-1)} \beta^{2} \\
-\frac{\overline{\mathrm{b}}}{(\gamma-1)}-\frac{1}{\mathrm{M}^{2}(\gamma-1)}-\frac{1}{2}
\end{array}\right]
\end{gathered}
$$

Because of the dependence of the equations (3.12) to (3.5), (3.20) and (3.21) on $\bar{b}$, similarity solution exists only when $\overline{\mathrm{b}}$ is a constant, that is only when the initial density $\rho_{1}$ is constant. The problem with the flow of a non-ideal gas is different from that of the perfect gas problem. In the latter case, similarity solution exists for initial density varying as some power of distance (Vishwakarma et al[7], Elliott[1], Christer and Helliwell[25], and Purohit[39]). But, it is not true for the problem with the flow of a non-ideal gas.

In addition to the shock conditions (3.21), the condition to be satisfied at the inner boundary surface is that the velocity of the fluid is equal to the velocity of inner boundary itself. This kinematic condition, from equations (3.1) and (3.12), can be written as

$$
\mathrm{U}\left(\eta_{\mathrm{p}}\right)=\eta_{\mathrm{p}}
$$

Now, the equations (3.12) to (3.15) and (3.20) may be integrated, numerically, with the boundary conditions (3.21) and the appropriate values of the constant parameters $\gamma, \bar{b}, \alpha, \omega, M^{2}, M_{A}^{2}$ and $N$ to obtain $D, H, P, Q$, and $U$. 
For exhibiting the numerical solutions, it is convenient to write the flow-variables in the following non-dimensional form as

$$
\begin{aligned}
\frac{\mathrm{u}}{\mathrm{u}_{2}} & =\frac{\mathrm{U}(\eta)}{\mathrm{U}(1)}, \\
\frac{\rho}{\rho_{2}} & =\frac{\mathrm{D}(\eta)}{\mathrm{D}(1)}, \\
\frac{\mathrm{p}}{\mathrm{p}_{2}} & =\frac{\mathrm{P}(\eta)}{\mathrm{P}(1)}, \\
\frac{\mathrm{h}}{\mathrm{h}_{2}} & =\frac{\mathrm{H}(\eta)}{\mathrm{H}(1)}, \\
\frac{\mathrm{q}}{\mathrm{q}_{2}} & =\frac{\mathrm{Q}(\eta)}{\mathrm{Q}(1)}
\end{aligned}
$$

\section{Results and Discussion}

The non-dimensional flow variables $\mathrm{u} / \mathrm{u}_{2}, \rho / \rho_{2}$, $\mathrm{p} / \mathrm{p}_{2}, \mathrm{~h} / \mathrm{h}_{2}$ and $\mathrm{q} / \mathrm{q}_{2}$ are obtained by numerical integration of the equations (3.12) to (3.15) and (3.20) with the boundary conditions (3.21). For the purpose of numerical calculations, the values of constant parameters are taken as (Roberts and $\mathrm{Wu}[17]$, Elliott[1], Singh and Mishra[6], Rosenau[29]): $\gamma=1.4 ; \bar{b}=0.0,0.05,0.1 ; \alpha=-2 ; \omega=1,1.2$; $\mathrm{M}^{2}=20 ; \mathrm{M}_{\mathrm{A}}^{2}=10$ and $\mathrm{N}=10,100$. The Value $\overline{\mathrm{b}}=0$ corresponds to the case of a perfect gas. Figures 1-5 show the variation of the flows variables $\mathrm{u} / \mathrm{u}_{2}, \rho / \rho_{2}, \mathrm{~h} / \mathrm{h}_{2}, \mathrm{p} / \mathrm{p}_{2}$, and $\mathrm{q} / \mathrm{q}_{2}$ with $\eta$ at various values of the parameters $\overline{\mathrm{b}}$, $\alpha, N, M^{2}, M_{A}^{2}, \omega, \gamma$. It is shown that, as we move inward from the shock front towards the inner contact surface, the reduced magnetic field $h / h_{2}$ and the reduced radiation heat flux $\mathrm{q} / \mathrm{q}_{2}$ increase and the reduced pressure $\rho / \rho_{2}$ decreases. Also, as we move inwards from the shock surface, the reduced velocity $\mathrm{u} / \mathrm{u}_{2}$ increases, and starts to decrease after attaining a maximum near the inner contact surface. The reduced density $\rho / \rho_{2}$ decreases from the shock front to the inner contact surface in all the cases except in the case $\overline{\mathrm{b}}=0, \mathrm{~N}=10, \omega=1$, where it increases and starts to decrease after attaining a maximum.

The effects of an increase in the value of the parameter of non-idealness of the gas $\bar{b}$ are (from table 1 and figures $1-5)$

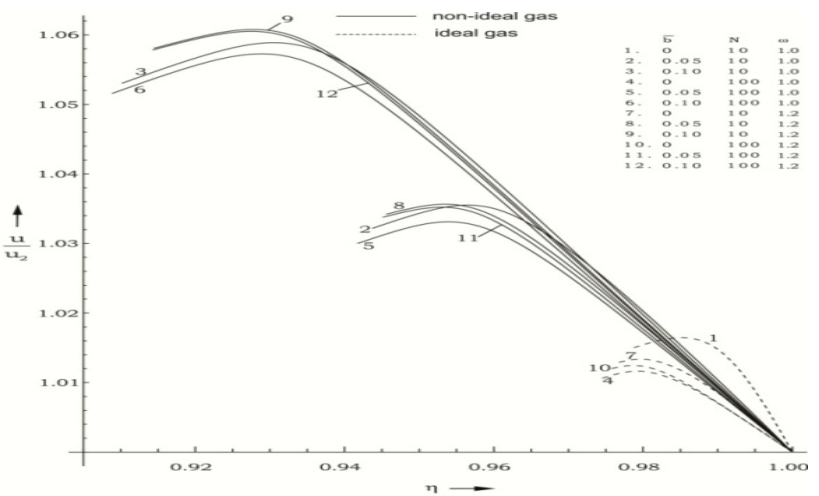

Figure 1. variation of non-dimensional velocity $\frac{u}{u_{2}}$ with non-dimensional distance $\eta$

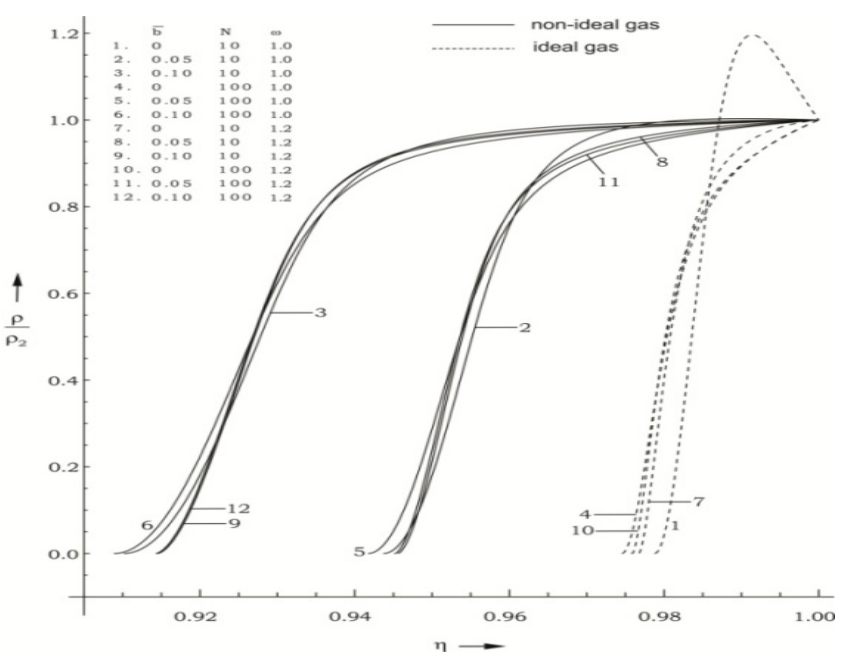

Figure 2. variation of non-dimensional density $\frac{\rho}{\rho_{2}}$ with non-dimensional distance $\eta$

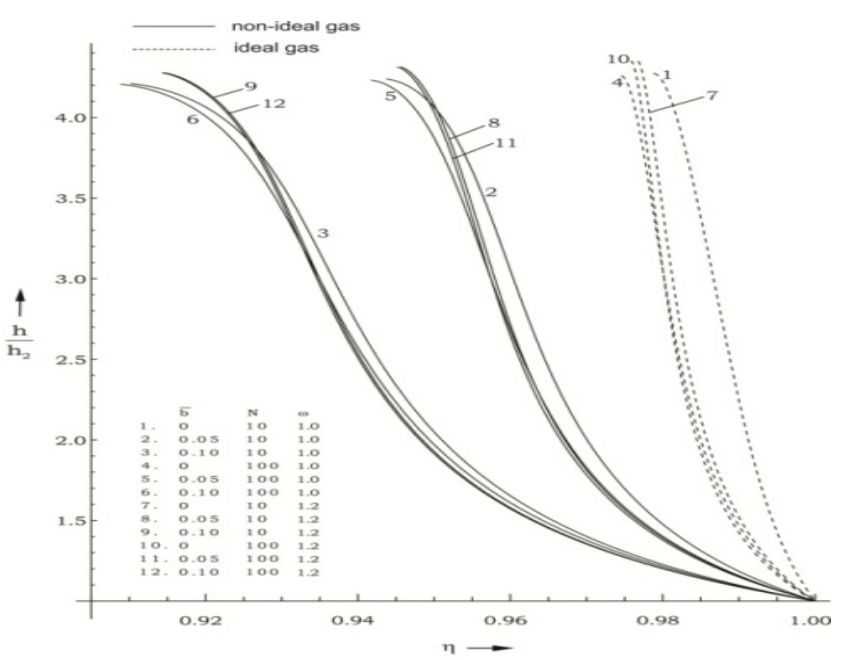

Figure 3. variation of non-dimensional magnetic field $\frac{h}{h_{2}}$ with non-dimensional distance $\eta$

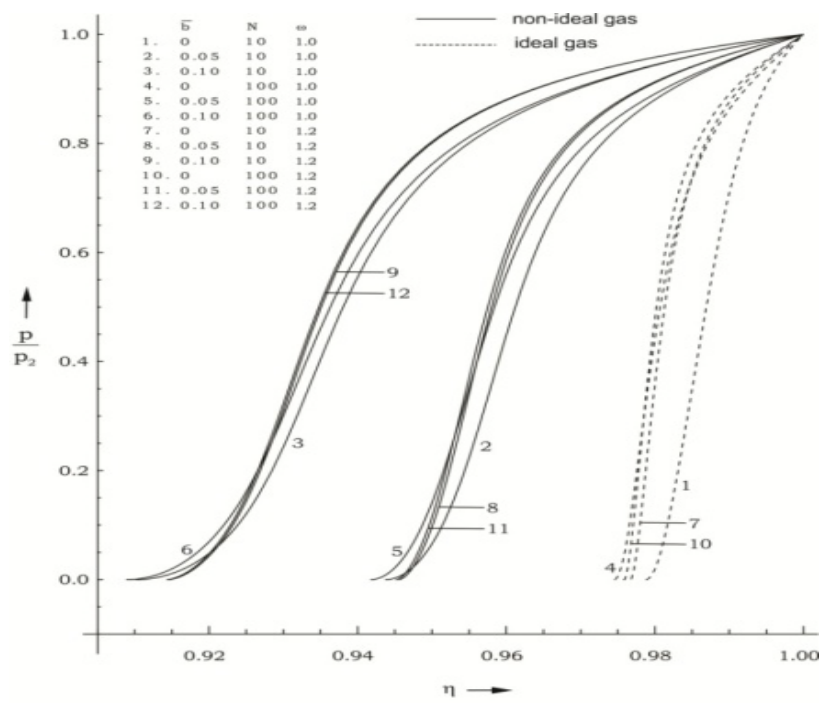

Figure 4. variation of non-dimensional pressure $\frac{p}{p_{2}}$ with non-dimensional distance $\eta$ 


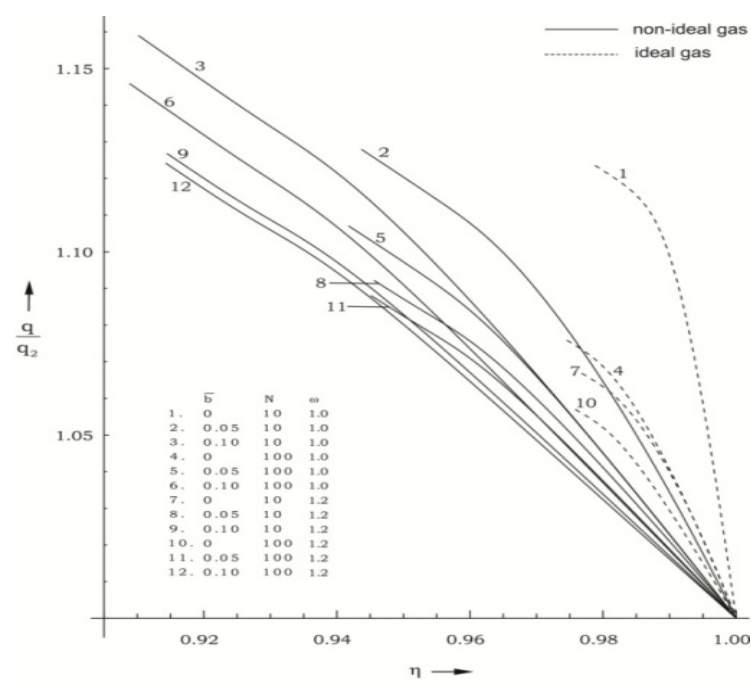

Figure 5. variation of non-dimensional radiation flux $\frac{\mathrm{q}}{\mathrm{q}_{2}}$ with non-dimensional distance $\eta$

(i). to increase the distance of the inner expanding surface from the shock front, i.e. to decrease the shock strength;

(ii). to increase the value of $\beta$, i.e. to decrease the shock strength. This is the same as concluded in (i) above. Therefore, the non-idealness of the gas has decaying effect on the shock wave.

(iii). to increase the reduced pressure $\mathrm{p} / \mathrm{p}_{2}$;

(iv). to increase the reduced velocity $\mathrm{u} / \mathrm{u}_{2}$ and reduced density $\rho / \rho_{2}$, in general; and

(v). to decrease the reduced magnetic field $\mathrm{h} / \mathrm{h}_{2}$ and the reduced heat flux $\mathrm{q} / \mathrm{q}_{2}$.

The effects of an increase in the value of radiation parameter $\mathrm{N}$ are

(i). to decrease the reduced velocity $\mathrm{u} / \mathrm{u}_{2}$ and reduced radiation heat flux $\mathrm{q} / \mathrm{q}_{2}$ (see figures 1 and 5);

(ii). to increase the reduced pressure $\mathrm{p} / \mathrm{p}_{2}$ (figure 4); and

(iii). to decrease the value of $\eta_{p}$ (table 1), i.e. to decrease

the shock strength. This decrease in the shock strength is due to the fact that the transport of energy through radiation is faster at higher values of $\mathrm{N}$.

The effects of an increase in the value of the exponent in the law for energy input $\omega$ (or the exponent in the law for initial magnetic field $n$ ) are

(i). to decrease the reduced magnetic field $h / h_{2}$ and the reduced radiation heat-flux $\mathrm{q} / \mathrm{q}_{2}$, but the effect is small when $\mathrm{N}=100$ (see the figure 3 and 5);

(ii). to increase the reduced pressure $\mathrm{p} / \mathrm{p}_{2}$, but the effect is small when $\mathrm{N}=100$ (see figure 4);

(iii). to decrease the reduced velocity $\mathrm{u} / \mathrm{u}_{2}$ when $\mathrm{N}=10$, but to increase it when $\mathrm{N}=100$ (see figure 1). The above results ((i), (ii), (iii)) show that the effects of an increase in $\omega$ are reduced or reversed by an increase in the radiation parameter $\mathrm{N}$; and

(iv). to increase the value of $\eta_{p}$ (see table 1), in general, i.e. to decrease the distance between the inner contact surface and the shock front. This shows that an increase in the value of $\omega$ increases the shock strength. This is due to the fact that an increase in the value of $\omega$ increases the rate of energy input to the flow between the inner contact surface and the shock front.

Table 1. Position of the inner boundary surface $\eta_{p}$, and the density ratio across the shock front $\beta\left(=\rho_{1} / \rho_{2}\right)$ for $\gamma=1.4, \alpha=-2, M^{2}=20$, $\mathrm{M}_{\mathrm{A}}^{2}=10$, and different values of $\overline{\mathrm{b}}, \omega, \mathrm{N}$

\begin{tabular}{|c|c|c|c|c|c|}
\hline \multirow{3}{*}{$\overline{\mathrm{b}}$} & \multicolumn{3}{|c|}{$\eta_{\mathrm{p}}$} & \multirow{2}{*}{$\beta$} \\
\cline { 2 - 5 } & \multicolumn{2}{|c|}{$\omega=1.0$} & \multicolumn{2}{|c|}{$\omega=1.2$} & \multirow{2}{*}{$\beta$} \\
\cline { 2 - 5 } & $\mathrm{N}=10$ & $\mathrm{~N}=100$ & $\mathrm{~N}=10$ & $\mathrm{~N}=100$ & \\
\hline 0.00 & 0.978780 & 0.974554 & 0.976776 & 0.975816 & 0.0357143 \\
\hline 0.05 & 0.943699 & 0.941726 & 0.945589 & 0.945115 & 0.0857143 \\
\hline 0.10 & 0.910155 & 0.908845 & 0.914409 & 0.914288 & 0.1357140 \\
\hline
\end{tabular}

\section{Conclusions}

In the present work, similarity solutions are obtained for the flow field behind a gas-ionizing cylindrical shock wave propagating in a non-ideal gas in presence of an azimuthal magnetic field, with radiation heat-flux and time dependent energy input. It is observed that as the shock propagates, its velocity $\mathrm{V}$ decreases with the ambient magnetic field $h_{1}$. It is investigated that an increase in the parameter of non-idealness of the gas $\bar{b}$, or in the exponent for energy input $\omega$, or in the radiation parameter $\mathrm{N}$ modifies the distribution of the flow variables behind the shock, and the non-idealness of the gas or the presence of the radiation heat-flux decays the shock wave. It is also investigated that the effects of an increase in the rate of energy input (i.e. an increase in $\omega)$ are reduced or reversed by an increase in the radiation parameter $\mathrm{N}$.

\section{REFERENCES}

[1] Elliott, L. A., Similarity methods in radiation hydrodynamics, Proc. Roy. Soc. Lond., 1960, 258, 287-301

[2] Wang, K. C., The piston problem with thermal radiation, J. Fluid Mech., 1964, 20, 447-457

[3] Helliwell, J. B., Self-similar piston problems with radiative heat transfer, J. Fluid Mech., 1969, 37, 497-512

[4] Ni Castro J. R., Similarity analysis of the radiative gas-dynamic equations with spherical symmetry, Phys. Fluids., 1970, 13, 2000-2006

[5] Ghoniem, A. F., Kamel, M. M., Berger, S. A., and Oppenheim, A. K., Effects of internal heat transfer on the structure of self-similar blast waves, J. Fluid Mech., 1982, 117, 473-491

[6] Singh, J. B., and Mishra, S. K., Modelling of self-similar cylindrical shock wave in radiation-magnetogasdynamics, Astrophys. Space Sci., 1986, 127, 33-43

[7] Vishwakarma, J. P., Srivastava, R. C., and Kumar, A., An exact similarity solution in radiation-magnetogasdynamics 
for the flows behind a spherical shock", Astrophys. Space Sci., 1987, 129, 45-52

[8] Singh, L. P., Sharma, V. D., and Ram, R., Flow Pattern induced by a piston impulsively moving in a perfectly conducting inviscid radiating gas, Phys. Fluids 1989, 3, 692-699

[9] Ganguly, A., and Jana, M., Propagation of a shock wave in self-gravitating, radiativemagnetohydrodynamic non- uniform rotating atmosphere", Bull. Cal. Math. Soc., 1998, 90, $77-82$

[10] Anisimov, S. I., and Spiner, O. M., Motion of an almost ideal gas in the presence of a strong point explosion, J. Appl. Math. Mech., 1972, 36, 883-887

[11] Rangarao M. P., and Purohit N. K., Self-similar piston problem in non-ideal gas, Int. J. Eng. Sci., 1976, 1491-1497

[12] Wu, C. C., and Roberts, P. H., Shock-wave propagation in a sonoluminescing gas bubble, Phys. Rev. Lett., 1993, 70, 3424-3427

[13] Madhumita, G., and Sharma, V. D., Imploding cylindrical and spherical shock waves in a non-ideal medium, J. Hyperbolic Differ. Equ., 2004, 1:521-530

[14] Arora, R., and Sharma, V. D., Convergence of strong shock in a van der Waals gas, SIAM J. Appl Math., 2006, 66: $1825-1837$

[15] Vishwakarma, J. P., and Nath, G., Similarity solutions for unsteady flow behind an exponential shock in a dusty gas, Phys. Scr., 2006, 74, 493-8

[16] Vishwakarma, J. P., and Nath, G., Self-similar solution of a shock propagation in a mixture of a non-ideal gas and small solid particles, Meccanica, 2009, 44, 239-54

[17] Roberts, P. H., and Wu, C. C., Structure and stability of a spherical implosion, Phys. Lett., 1996, 213, 59-64

[18] Roberts, P. H., and Wu, C. C., The shock-wave theory of sonoluminescene, in Shock Focussing Effect in Medical Science and Sonoluminescene (Eds. R. C. Srivastava, D. Leutloff, K. Takayama, H. Groning), Springer Verlag, 2003

[19] Vishwakarma, J. P., Maurya, A. K., and Singh, K. K., Self-similar adiabatic flow headed by magnetogasdynamics cylindrical shock wave in a rotating non-ideal gas, Geophys. Astrophys. Fluid Dyn., 2007, 101, 115-167

[20] Greenspan, H. P., Similarity solution for a cylindrical shock-magnetic field interaction, Phys. Fluids, 1962, 5, 255-259

[21] Greifinger, C., and Cole, J. D., Similarity solution for cylindrical magnetohydrodynamic ( MHD ) blast waves, Phys. of Fluids, 1962, 5, 1597-1607

[22] Christer, A. H., Self-similar cylindrical ionizing shock and detonation waves, Z. Angew Math. Mech, 1972, 52, 11-22
[23] Rangarao, M. P., and Ramana, B. V., Self-similar cylindrical magnetogasdynamic and Ionizing shock waves, 1973, 11, $337-351$

[24] Singh, J. B., Self-similar cylindrical Ionizing shock waves with radiation heat flux, Indian J. Tech., 1983, 21, 315-318

[25] Christer, A. H., and Helliwell, J. B., Cylindrical shock and detonation waves in magnetogasdynamics, J.Fluid Mech. $1969,39,705-725$

[26] Vishwakarma, J. P., and Pandey, S. N., Converging cylindrical shock waves in a non-ideal gas with an axial magnetic field, Def. Sci. J., 2004, 56, 721-731

[27] Gretler, W., and Wehle, P., Propagation of blast waves with exponential heat release and internal heat conduction and thermal radiation, Shock Wave; 1993, 3, 95-104

[28] Pomraning, G. C., The equations of radiation Hydrodynamics, Int. Ser. Monographs in Natural Philosophy 54,Pergamon Press, New York, 1973

[29] Rosenau, P., Equatorial propagation of axisymmetric magnetohydrodynamic shock II, Phys. Fluids, 1977, 20, 1097-1103

[30] Laumbach, D. D., and Probstein, R. F., A point explosion in a cold exponential atmosphere, part 2 Radiating flow, J. Fluid Mech., 1970, 40, 833-858

[31] Singh, J. B., and Srivastava, S. K., Cylindrical ionizing shock waves in non-ideal gas, J. P. A. S. Jaunpur, 1991, 2, 39-46

[32] Zel'dovich, Ya. B., and Raizer, W. P., Physics of Shock Waves and High Temperature Hydrodynamic Phenomena, Vol. II, Academic Press, New York, 1967

[33] Rosenau, P., and Frankenthal, S., Shock disturbances in a thermally conducting solar wind Astrophys. J., 1976, 208, 633-637

[34] Rogers, M. H., Similarity flows behind strong shock waves. Quart. J. Mech. Appl. Math., 1958, XI (II), 411-422

[35] Freeman, R. A., Variable energy blast wave., J. Phys. D., $1968,1,1697-1710$

[36] Freeman, R. A., and Cragges, J. D., Problem of spark channel formations, Brit. J. Appl. Phys. ( J. Phys. D ), 1969, 2, 421-427

[37] Director, M. N., and Dabora, E. K., An experimental investigation of variable energy blast waves, ActaAstronautica, 1977, 4, 391-407

[38] Abdel-Raouf, A. M., and Gretlar, W., Quasi-similar solutions for blast wave with internal heat transfer effects, Fluid Dyn. Res., 1991, 8(5-6), 273-285

[39] Purohit, S. C., Self-similar homothermal flow of self-gravitating gas behind shock wave, J. Phys. Soc. Japan, $1974,36,288-292$ 